\title{
Update From the Field of Renal Sympathetic Denervation: A Focus on Safety Nomenclature Considerations
}

Therapeutic Innovation \& Regulatory Science 2017, Vol. 5I(6) 664-668 (C) DIA 2017

Reprints and permission: sagepub.com/journalsPermissions.nav DOI: $10.1177 / 2168479017739927$ tirs.sagepub.com
I recently had the good fortune to attend the European Society of Cardiology's 2017 Congress, held in Barcelona at the end of August, and to take a subsequent vacation there with my wife Karen. It was a poignant time to be in the city as the terrorist attack on Las Ramblas had occurred shortly beforehand. The people of Barcelona could not have been more welcoming to the 30,000 Congress attendees: I greatly admire their resilience, and thank them for their warm hospitality.

One of the high-profile events at the Congress was the presentation of initial results from the SPYRAL HTN-OFF MED trial: data from the first 80 participants enrolled in the study at 3 months were presented in a "late-breaking clinical trial session." SPYRAL HTN-OFF MED is a randomized, concurrently controlled trial investigating the interventional procedure of renal sympathetic denervation as a treatment for mild to moderate hypertension in participants not taking antihypertensive medications: the control group consists of participants, also not taking antihypertensive medications, who undergo a sham procedure that is as similar as possible to the denervation procedure without actual ablation of the renal nerves. As is often the case with presentations of high-profile trial results at major international meetings, a paper detailing the results was e-published in a high-profile journal to coincide with the presentation. ${ }^{1}$

I read the paper with great interest for two reasons. First, the efficacy results from this trial had been awaited with great anticipation by many scientists and physicians. Second, I had been critical of safety-related nomenclature used in a multitude of reviews and commentaries addressing earlier trials of renal sympathetic denervation, and also critical of efficacy claims made in the broad literature based on studies (SYMPLICITY HTN $-1^{2}$ and SYMPLICITY HTN-2 ${ }^{3}$ ) whose designs did not legitimately permit such claims. ${ }^{4,5}$ SPYRAL HTN-OFF MED's design ${ }^{6}$ had the inherent ability to permit the claims of efficacy made in this case, and I was pleased to see that the nomenclature used in the reporting of safety issues was conservative and appropriate. After first providing some context, this Editorial summarizes the results presented, and also represents a plea for those writing (or about to write) journal articles addressing the latest developments in this field to avoid using the single, emphatic descriptor "safe."

Hypertension has been authoritatively identified as one of the largest contributors to the global burden of disease. ${ }^{7-9}$ It affects approximately 1 billion individuals worldwide, and continues to be among the most prevalent conditions seen in adult primary clinical care. ${ }^{10}$ It is arguably the most critical cardiovascular and cerebrovascular disease risk factor, and is ultimately a leading cause of death, stroke, myocardial infarction, and congestive heart failure. Considerable attention has therefore focused for multiple decades on improving the prevention and treatment of hypertension and cardiovascular disease by lifestyle modifications and pharmaceutical therapy, but it remains the case that an undesirably large percentage of individuals do not attain target blood pressure levels. (Detailed discussion of the reasons for this, including patient lack of adherence to appropriately prescribed exercise and medication regimens, less-than-optimal medication regimens prescribed by physicians who are not hypertension specialists, and lessthan-optimal access to hypertension specialists, is beyond the scope of this Editorial: references are provided for further reading. ${ }^{11-13}$ )

Resistant hypertension, which has been defined as blood pressure remaining above target levels despite the use of 3 or more antihypertensive medications at maximally prescribed doses (ideally, one being a diuretic) or blood pressure requiring 4 or more drugs to achieve control, ${ }^{14}$ can be particularly challenging pharmacologically. ${ }^{15,16}$ In the past decade, therefore, the interventional technique of renal sympathetic denervation was initially discussed as a purported treatment specifically for resistant hypertension, although it is now being discussed as a purported treatment for less severe forms of hypertension as well.

Renal sympathetic denervation is a procedure in which radiofrequency energy can be applied with an endovascular catheter inserted via the femoral artery to both renal arteries sequentially, with the resultant ablation of the sympathetic nerve fibers: alternate methods include ablation via ultrasound energy or injection of pharmaceutical agents into the perivascular space around the renal nerves. The sympathetic nervous system was brought to public awareness in the early part of the 20th century by Walter Cannon as a result of his research on the concept of the "fight or flight response" to stress. More recently, aberrations in this biological system have been found to be important in various clinical conditions, including hypertension and heart failure: in both disease states, there is "persistent and adverse activation of sympathetic outflows to the heart and kidneys." 17 Sympathetic innervation of the kidneys plays an important role in the pathogenesis of hypertension via modulation of renin secretion, glomerular filtration rate, and renal absorption of sodium. ${ }^{18}$ Decreasing such 
innervation as a potential treatment for hypertension is therefore physiologically plausible.

SYMPLICITY HTN-1 and SYMPLICITY HTN-2 have been discussed in some detail in a previous article in this journal. ${ }^{4}$ The subsequent SYMPLICITY HTN-3 was a "pivotal study designed as a prospective, randomized, masked procedure, single-blind trial evaluating the safety and effectiveness of catheter-based bilateral renal denervation for the treatment of uncontrolled hypertension despite compliance with at least 3 antihypertensive medications of different classes (at least one of which is a diuretic) at maximal tolerable doses." ${ }^{\prime 19}$ Approximately 530 participants were randomized into one of 2 treatment groups: the renal sympathetic denervation group, or a control treatment group experienced a sham procedure (renal angiography). The primary efficacy endpoint was change in office systolic blood pressure at 6 months. The mean change in the renal sympathetic denervation treatment group was $14.13 \mathrm{mmHg}$, and for the sham control treatment group, the mean change was $-11.74 \mathrm{mmHg}$, yielding a point estimate of the treatment effect of $-2.39 \mathrm{mmHg}$ : this difference was not statistically significant. The authors concluded that "this blinded trial did not show a significant reduction of systolic blood pressure in patients with resistant hypertension 6 months after renal-artery denervation as compared with a sham control."19

Despite failing to meet its primary efficacy endpoint, many statements following the publication of the SYMPLICITY HTN-3 report emphasized renal sympathetic denervation's safety (the report itself utilized more circumspect language). As an example (with the citation deliberately being omitted since this was not the only one), consider the following statement from a publication in a major cardiovascular journal: "The SYMPLICITY HTN-3 randomized, blinded, shamcontrolled trial confirmed the safety of renal denervation (RDN), but did not meet its primary endpoint." Safety is actually an extremely difficult quality to quantify (some authors advocate for the term harms instead ${ }^{20}$ ). Lessons concerning nomenclature can be learned from new drug development. It is an unfortunate but immutable fact that no biologically active drug is free from the possibility of causing adverse reactions in certain individuals who are genetically and/or environmentally susceptible, a realization that emphasizes the supreme importance of benefit-risk estimations in regulatory approval decisions (made at the public health level) and decisions in clinical practice (made at the individual patient level). ${ }^{21}$ The US Food and Drug Administration's Sentinel Initiative addressed this issue in the broader scope of medical products $^{22}$ :

Although marketed medical products are required by federal law to be safe for their intended use, safety does not mean zero risk. A safe product is one that has acceptable risks, given the magnitude of benefit expected in a specific population and within the context of alternatives available.
Strictly speaking, claims in the literature that, while being shown to be ineffective (at the global group mean level) in SYMPLICITY HTN-3, the safety of the procedure of renal sympathetic denervation was confirmed skated on extremely thin ice: in the context of a lack of sufficient efficacy to meet a primary efficacy endpoint, any degree of risk - a rash or irritation at the catheter entry site in the groin region, for examplearguably leads immediately to an unfavorable benefit-risk balance.

\section{SPYRAL HTN-OFF MED}

Upon the collectively expressed "failure" (not my preferred choice of word) of SYMPLICITY HTN-3 to provide evidence of efficacy, teeth gnashing abounded. Fortunately, calmer scientific deliberation also surfaced and consensus publications suggested where and how to go next. ${ }^{23,24}$ SPYRAL HTNOFF MED emerged from such discussions.

SPYRAL HTN-OFF MED differed from SYMPLICITY HTN-3 in various ways. First, the population from which participants were enrolled had less severe hypertension, and participants did not take antihypertensive medications during the 12-week period from procedure to postprocedure assessment. Second, the renal denervation catheter was different from that used in the SYMPLICITY trials (a single electrode): this time a multielectrode catheter was employed that delivered "up to four simultaneous, radiofrequency ablations in a helical pattern and included branch vessel treatment" as well as in treatment in the main renal arteries. ${ }^{1}$

Third, the design of SPYRAL HTN-OFF MED ${ }^{6}$ benefited from accumulating insights and the considered opinion of authoritative individuals presented in a Scientific Statement from the American Society of Hypertension. ${ }^{24}$ The study was designed as a small, multicenter, prospective, international, single-blind, randomized, sham-controlled, proof-of-concept trial involving participants with an office systolic blood pressure of $150 \mathrm{mmHg}$ or greater and less than $180 \mathrm{mmHg}$, office diastolic blood pressure of $90 \mathrm{mmHg}$ or greater, and a mean 24-hour ambulatory systolic blood pressure of $140 \mathrm{mmHg}$ or greater and less than $170 \mathrm{mmHg}$ at second screening. Participants, who were drug-naïve or had discontinued their antihypertensive medications (hence the term "OFF MED"), underwent renal angiography and were randomly assigned to renal denervation or sham control. The primary endpoint was change in 24-hour blood pressure at the end of month 3 rather than office pressure (see Turner and O'Brien ${ }^{25}$ for discussion of the importance of such blood pressure assessment).

The trial's blood pressure level inclusion criteria and 12-week duration were closely related. In the context of an important research question, it has been considered acceptable that certain participants with these levels of blood pressure (those without moderately severe or severe kidney disease, and with a lack of any cardiovascular or neurologic event within the past 6 months) can be untreated with antihypertensive 
Table I. Mean Changes in 24-Hour Blood Pressure at 3 Months.

\begin{tabular}{lccc}
\hline Parameter $(\mathrm{mmHg})$ & $\begin{array}{c}\text { Renal } \\
\text { Denervation } \\
\text { Group }\end{array}$ & $\begin{array}{c}\text { Sham } \\
\text { Control } \\
\text { Group }\end{array}$ & $\begin{array}{c}\text { Treatment } \\
\text { Effect Point } \\
\text { Estimate }^{\mathrm{a}}\end{array}$ \\
\hline Systolic blood pressure & -5.5 & -0.5 & -5.0 \\
Diastolic blood pressure & -4.8 & -0.4 & -4.4 \\
\hline
\end{tabular}

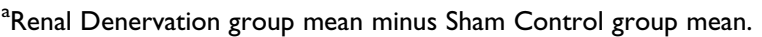

medications for up to 12 weeks. ${ }^{24}$ Such a phase II proof-ofconcept design represents a relatively quick and efficient method of determining a signal of effectiveness before commencing a complex pivotal Phase III trial.

The efficacy results are presented in Table 1. They showed a statistically significant reduction in 24-hour ambulatory systolic and diastolic blood pressures at 12 weeks in participants with mild to moderate hypertension following renal denervation not receiving antihypertensive medications that was not observed in the sham control group (the same was true for office blood pressures). The authors concluded that these results provide proof-of-concept of catheter-based renal denervation to reduce blood pressure in these circumstances. ${ }^{1}$

\section{Safety Information from SPYRAL HTN-OFF MED}

A key statement in the trial's report reads as follows: "There were no major safety events in either group despite the absence of pharmacological therapy from enrolment to the 3-month follow-up and a more aggressive renal denervation procedure than that of previous trials in this setting, extending into renal artery branch vessels." "The authors are to be commended for using this language, not simply stating categorically that the procedure is safe.

Delving deeper into the Appendix provided along with the online version of their paper provides more detailed information. Nine safety endpoints were reported at 3 months: new myocardial infarction, major bleeding (using the Thrombolysis in Myocardial Infarction [TIMI] definition of intracranial hemorrhage, $\geq 5 \mathrm{~g} / \mathrm{dL}$ decrease in hemoglobin concentration, $\geq 15 \%$ absolute decrease in hematocrit, or death due to bleeding within 7 days of the procedure), new-onset end-stage renal disease, serum creatinine elevation $>50 \%$, significant embolic event resulting in end-organ damage, vascular complications, hospitalization for hypertensive crises/emergency, and new stroke. There were zero occurrences of these endpoints in both the sham-procedure group $(\mathrm{n}=42)$ and the renal denervation group $(\mathrm{n}=38)$. While not explicitly called safety endpoints, the authors also reported baseline values and changes at 3 months in 7 laboratory values: plasma renin activity, aldosterone, serum creatinine, estimated glomerular filtration rate, glucose, potassium, and sodium. None of the mean changes at 3 months between the two groups approached statistical significance.
Having acknowledged earlier in this Editorial that SPYRAL HTN-OFF MED was designed in accordance with recommendations made and a Scientific Statement from the American Society of Hypertension, it would be inappropriate to make a big song and dance about there "only" being 38 participants in the renal denervation group, and hence that safety data are "only" available for 38 individuals. That said, it remains a very small number: while this trial falls on a corrected pathway compared with previous trials, the authors know that there is still a long way to travel on that path for evaluations of efficacy as well as safety: see also Azizi. ${ }^{26}$

\section{A Plea to Authors Preparing Commentaries about SPYRAL HTN-OFF MED}

When people write commentaries about the SPYRAL HTNOFF MED trial, I hope they will refrain from saying it showed that renal sympathetic denervation is "safe." The authors did not use this terminology, so why should others assign it to them?

Safe is sitting comfortably in your favorite armchair drinking a nice cup of hot tea (or a nice glass of iced tea) watching a rerun episode of The Golden Girls (a delightfully life-affirming US television situation-comedy show that originally ran between 1985 and 1992). Safe, that is, until you laugh so hard you spill some tea on your shirt or blouse. Oh well, a small price to pay for watching a great show-it can go in the next load of laundry.

Safe is smiling self-assuredly while serving warm cake and ice-cold lemonade to all the excited attendees at your daughter's fifth birthday party in your bucolic backyard on a lazy summer's afternoon. Safe, that is, until your neighbors' child and daughter's best friend accidentally trips over a bunch of balloons while running, empty plate in hand, for a second serving of cake, hitting her nose on the ground and bleeding on her brand new fashionable and expensive party dress purchased just that morning, and you receive a discreetlydelivered but nonetheless biting tongue-lashing from your daughter's best friend's apoplectic parents for your brazen and unforgivable dereliction of child-minding duties. Oh well, you take solace in your well-received offer to replace the brand-new fashionable and expensive party dress with one just like it, and great pleasure that your daughter will always have the memories of cake, lemonade, and lots of party guests at her house that day.

Safe is being about to toast your loved one with chilled champagne while sitting together on your holiday home's patio watching the sun set over the magnificent Mediterranean sea on a mellow early September evening. Safe, that is, until the sound of the cork explosively leaving the bottle terrifies your sensitive and company-seeking new kitten, who, in chasing into the house for shelter, accidentally bumps into your doting dog, who in turn accidentally knocks over your antique coffee table, an event which in turn damages the cherished photograph sitting upon it that was taken while you watched a similarly 
sensational sunset together ten years ago to the day. Oh well, it can be repaired, and it can now be accompanied in the future by another photograph to be taken today on your 10th Anniversary: you wouldn't have missed the whole experience for the world.

Life is not $100 \%$ without risks (some greater than others), and it is an irrational expectation that any interventional procedure (or drug) should be $100 \%$ without risk, that is, unambiguously "safe." That said, given that SPYRAL HTN-OFF MED provided evidence of a certain degree of efficacy, it is now permissible to move to discussions of a potentially favorable benefit-risk balance. First, longer-term assessments of efficacy and safety are needed (and in the case of safety, likely a much broader set of parameters than the major events discussed in the current report of the trial). Another centrally important factor will be endeavoring to determine individual patients who, more than others, are likely to benefit or likely not to benefit from the procedure: the latter may turn out to be easier than the former since specific and readily identifiable contraindications may be more readily identified.

If additional compelling evidence of efficacy is obtained, and compelling evidence also suggests minimal risk in the long term, it may be possible to consider language such as this: Renal sympathetic denervation is an interventional procedure associated with minimal risk, and for patients considered likely to experience therapeutic benefit of a certain magnitude - and who also do not have specific contraindications - it may have a favorable benefit-risk balance when considered in the context of other potential treatments.

Let's see what happens in the next couple of years.

\section{J. Rick Turner, PhD, DSc, FASH, FACC, FESC Editor-in-Chief, TIRS}

\section{References}

1. Townsend RR, Mahfoud F, Kandzari DE, et al; SPYRAL HTNOFF MED Trial Investigators. Catheter-based renal denervation in patients with uncontrolled hypertension in the absence of antihypertensive medications (SPYRAL HTN-OFF MED): a randomised, sham-controlled, proof-of-concept trial. Lancet. 2017 Aug 28 [Epub ahead of print]

2. Krum H, Schlaich M, Whitbourn R, et al. Catheter-based renal sympathetic denervation for resistant hypertension: a multicenter safety and proof-of-principle cohort study. Lancet. 2009;373: 1275-1281.

3. Esler MD, Krum H, Sobotka PA, et al. Symplicity HTN-2 Investigators. Renal sympathetic denervation in patients with treatment resistant hypertension (the Symplicity HTN-2 Trial): a randomised controlled trial. Lancet. 2010;376:1903-1909.

4. Turner JR, O'Brien E. Inferences beyond a study design's grasp: a cautionary case study from the recent renal sympathetic denervation. Therapeutic Innovation \& Regulatory Science. 2015;49:86-92.
5. Lee J, Turner JR. Raising the bar in renal sympathetic denervation research and reporting. J Clin Hypertens (Greenwich). 2016;18: 89-94.

6. Kandzari DE, Kario K, Mahfoud F, et al. The SPYRAL HTN Global Clinical Trial Program: Rationale and design for studies of renal denervation in the absence (SPYRAL HTN OFF-MED) and presence (SPYRAL HTN ON-MED) of antihypertensive medications. Am Heart J. 2016;171:82-91.

7. Horton R. GBD 2010: understanding disease, injury, and risk. Lancet. 2013;380:2053-2054.

8. Das P, Samarasekera U. The story of GBD 2010: a "super-human" effort. Lancet. 2013;380:2067-2070.

9. Lim SS, Vos T, Flaxman AD, et al. A comparative risk assessment of burden of disease and injury attributable to 67 risk factors and risk factor clusters in 21 regions, 1990-2010: a systematic analysis for the Global Burden of Disease Study 2010. Lancet. 2013;380: 2224-2260.

10. Turner JR, Viera AJ, Shimbo D. Ambulatory blood pressure monitoring in clinical practice: a review. Am J Med. 2015;128:14-20.

11. Turner JR. Patient and physician adherence in hypertension management. J Clin Hypertens (Greenwich). 2013;15:447-452.

12. White WB, Turner JR, Sica D, et al. Scientific statement. Detection, evaluation, and treatment of severe and resistant hypertension: proceedings from an American Society of Hypertension Interactive Forum held in Bethesda, Maryland, USA, October 10th 2013. J Am Soc Hypertens. 2014;8:743-757.

13. Burnier M. Drug adherence in hypertension [published online ahead of print September 1, 2017]. Pharmacol Res.

14. Judd E, Clahoun DA. Apparent and true resistant hypertension: definition, prevalence and outcomes. J Hum Hypertens. 2014;28: 463-468.

15. Cai A, Calhoun DA. Resistant hypertension: an update of experimental and clinical findings. Hypertension. 2017;70:5-9.

16. Cai A, Feng Y, Zhou Y. A comprehensive review of an unmet public health issue: resistant hypertension. Clin Exp Hypertens. 2017;39:101-107.

17. Parati G, Esler M. The human sympathetic nervous system: its relevance in hypertension and heart failure. Eur Heart J. 2012; 33: 1058-1066.

18. Gulati V, White WB. Review of the state of renal nerve ablation for patients with severe and resistant hypertension. J Am Soc Hypertens. 2013;7:484-493.

19. Bhatt DL, Kandzari DE, O'Neill WW, et al. SYMPLICITY HTN-3 Investigators. A controlled trial of renal denervation for resistant hypertension. N Engl J Med. 2014;370:1393-1401.

20. Ioannidis JP, Evans SJ, Gøtzsche PC, et al; CONSORT Group. Better reporting of harms in randomized trials: an extension of the CONSORT statement. Ann Intern Med. 2004;141:781-788.

21. Turner JR, Karnad DR, Kothari S. Cardiovascular Safety in Drug Development and Therapeutic Use: New Methodologies and Evolving Regulatory Landscapes. Gewerbestrasse, Switzerland:, 2017.

22. FDA. The Sentinel Initiative: National Strategy for Monitoring Medical Product Safety. https://www.fda.gov/downloads/Safety/ 
FDAsSentinelInitiative/UCM124701.pdf. Published May 2008. Accessed September 25, 2017.

23. Weber MA, Kirtane A, Mauri L, et al. Renal denervation for the treatment of hypertension: making a new start, getting it right. $J$ Clin Hypertens (Greenwich). 2015;17:743-750.

24. White WB, Galis Z, Henegar J, et al. Device therapy for hypertension: pathways for moving development forward. Proceedings from an American Society of Hypertension Interactive Forum held in Bethesda, Maryland, USA, June 26th 2014. J Am Soc Hypertens. 2015;9: 341-350.

25. Turner JR, O'Brien E. Diagnosis and treatment of resistant hypertension: the critical role of ambulatory blood pressure monitoring. J Clin Hypertens (Greenwich). 2013;15:868-873.

26. Azizi M. Catheter-based renal denervation for treatment of hypertension [published online ahead of print August 28, 2017]. Lancet. [Epub ahead of print] 